\title{
RSNT-cFastI CA for Complex-Valued Noncircular Signals in Wireless Sensor Networks
}

\author{
Changliang Deng ${ }^{1}$, Yimin Wei ${ }^{1}$, Yuehong Shen ${ }^{1}$, Wei Zhao ${ }^{1}$ and Hongjun $\mathrm{Li}^{2}$ \\ ${ }^{1}$ College of Communications Engineering, The Army Engineering University of PLA \\ 88 Houbiaoying Rd., Qinhuai District, Nanjing, 210007 China \\ ${ }^{2}$ National Innovation Institute of Defense Technology, Academy of Military Science \\ 53 Dongdajie Rd., Fengtai District, Beijing, 100071, China \\ [e-mail: haishenbo@163.com, wym73@163.com, chunfeng22259@163.com,gmajyie@126.com, \\ lihongjun86@126.com] \\ *Corresponding author: Changliang Deng
}

Received January 8, 2018; revised March 21, 2018; accepted April 6, 2018; published October 31, 2018

\begin{abstract}
This paper presents an architecture for wireless sensor networks (WSNs) with blind source separation (BSS) applied to retrieve the received mixing signals of the sink nodes first. The little-to-no need of prior knowledge about the source signals of the sink nodes in the BSS method is obviously advantageous for WSNs. The optimization problem of the BSS of multiple independent source signals with complex and noncircular distributions from observed sensor nodes is considered and addressed. This paper applies Castella's reference-based scheme to Novey's negentropy-based algorithms, and then proposes a novel fast fixed-point (FastICA) algorithm, defined as the reference-signal negentropy complex FastICA (RSNT-cFastICA) for complex-valued noncircular-distribution source signals. The proposed method for the sink nodes is substantially more efficient than Novey's quasi-Newton algorithm in terms of computational speed under large numbers of samples, can effectively improve the power consumption effeciency of the sink nodes, and is significantly beneficial for WSNs and wireless communication networks (WCNs). The effectiveness and performance of the proposed method are validated and compared with three related BSS algorithms through theoretical analysis and simulations.
\end{abstract}

Keywords: wireless sensor networks, network architecture, complex fast independent component analysis, reference-signal negentropy cFastICA, noncircular signals, blind source separation

This research was supported by the National Natural Science Foundation of China under the grants of No. 6117206 61401506 and 6120242 and the China Postdoctoral Science Foundation under the grant of 2016M600349. 


\section{Introduction}

Wireless sensor networks (WSNs) are getting more and more concentration for their important theoretical and application values. One of the most important transmission strategy for the WSNs is for saving energy. Therefore, efficient collaborative signal processing algorithms that consume less energy for computation and communication are needed for the applications on WSNs.

\subsection{Literatures}

WSNs are composed of large number of sensor nodes that are densely deployed to their sink nodes to achieve signal sensing and transmitting, which is considered to be most important technology [1]. Recently, more and more works have been concentrated on wireless sensor networks because of its widely application to many important fields, such as industrial automation [2][3], agricultural modernization [4][5], space technology [6]-[8], information security [9], medical diagnosis [10], position location [11], transport [12], environment monitoring [13], and disaster warning [14]. Thus, the WSNs can be considered as the core components in achieving an overall sense of society and nature for human beings. Due to the ubiquitous and disorderly nature of electromagnetic signals, the sensing signals are interfered with by various noise sources and are difficult to distinguish. Thus, obviously, signal processing is very critical to the WSNs, as the sensing signals must be separated from complex-valued signals in the sink nodes [15]. The separation of multiple unknown sources from sensor nodes has been studied and applied in many areas such as the extraction of individual speech signals from a mixture of simultaneous speakers (the famous so-called 'cocktail party' problem), elimination of the cross interference between horizontally and vertically polarized microwaves in wireless communications and radar systems, and separation of multiple telephone signals at a base station and so on. However, WSNs are designed to be low cost and limited power, which is the greatest advantage, but is also the drawback because the sensing and signal separation algorithms have to consider firstly the power consumption problem[16]-[18].

Blind source separation (BSS) is such a method aimed at recovering unknown sources only from their observed data. Because it requires little or even no prior information or a mixing matrix, BSS has been widely applied in a variety of fields such as telecommunication [19][20], seismic exploration [21], biomedical detection [22]-[24], sensor networks [25]-[28], sensing compress [29]-[31], and machine diagnosis [32]-[38]. In recent decades, in the case of a linear multi-input multi-output (MIMO) instantaneous system, BSS has corresponded to independent component analysis (ICA), which is a widely recognized concept [15][39]. The core assumption in ICA can be reduced to the statistically mutual independence of sources. Complex BSS and ICA have applications of enormous potential such as in WSNs and wireless communication networks (WCNs). In these above-mentioned applications, most of the sources may be both sub-Gaussian and super-Gaussian and may have circular (rotationally invariant) or noncircular distributions, specifically in the complex domain [31]. Certain sub-Gaussian signals of engineering interest that are easily affected by noncircularity such as quadrature amplitude modulation (QAM), binary phase-shift keying (BPSK), uniform distributions, and complex sinusoids. In this paper, we mainly consider the ICA model of BSS in the WSNs in the case of complex noncircular sources, especially sub-Gaussian signals. 
Many solutions have been designed for performing complex-valued ICA with respect to noncircular distribution source signals. For example, in [40], Novey proposed gradientdescent and quasi-Newton algorithms for both circular and noncircular sources by utilizing analytic functions in a maximization of a non-Gaussianity framework. For noncircular sources that are especially sub-Gaussian, Novey's quasi-Newton algorithm performs well by using full second-order information conveyed through both covariance and pseudocovariance matrices. Recently, Castella designed a family of contrast functions referred to as reference-based functions in [41], which are essentially the cross-Cumulant or cross-Statistics between estimated output signals and the so-called reference signals. An appealing feature in common with these referencebased contrast criteria is that the corresponding optimization algorithms are quadratic with respect to the searched parameters.

\subsection{Motivation and contributions}

From the analysis above, it can be seen that WSN forms a natural platform for effective and low cost BSS. Thus, more effective algrithms for source separation is of necessity and emergency. In this paper, to further effectively address the optimization problem of the BSS of multiple independent source signals with complex and noncircular distributions in WCNs, we propose to apply Castellas reference-based scheme in Noveys negentropy-based algorithms. And then we propose a novel fast fixedpoint (FastICA) algorithm for complex-valued noncircular distribution source signal. In addition, for simplicity, the new algorithm is defined as reference-signal negentropy complex-ICA (RSNT-cFastICA). The main advantage of this paper is that an architecture of the WSNs with BSS is presented; the RSNT-cFastICA algorithm is applied for this architecture and is substantially more efficient than Novey's quasi-Newton algorithm [39] in terms of computational speed.

The main contributions of this paper are summarized as follows.

- The architecture of WSNs with BSS applied to solve the signal processing problems in the sink nodes is proposed first in this paper. And the architecture is also described and analyzed in detail. This will lead to our further study of the BSS problem for multiple WSNs.

- RSNT-cFastICA is proposed to solve the BSS problem for the sink nodes of WSNs. The mustability of RSNT-cFastICA is theoretically analyzed and proven.

- The effectiveness and performance of the proposed method are validated by comparing with three related BSS algorithms through simulations. This method is substantially more efficient than Novey's quasi-Newton algorithm in terms of computational speed with large numbers of samples, can effectively improve the power consumption effeciency by the sink nodes, and is significantly effective in WSNs and WCNs.

\subsection{Structure and notations}

The remainder of this paper is organized as follows. The architecture of the WSNs and the complex ICA model are shown in Section II. The local stability of the reference-based contrast criterion is described and proved in detail in Section III. The RSNT-cFastICA algorithm is derived and analyzed in Section IV. Simulation results are illustrated in Section V. Conclusions can be found in Section VI.

Notations: Bold lowercase and uppercase letters represent vectors and matrices. $(\cdot)^{T}$ and $(\cdot)^{H}$ denote the transpose and Hermitian transpose of vectors or matrices, respectively. $\mathbf{E}[\cdot]$ is the expectation operator. $\mathbf{R}^{u \times v}$ and $\mathbf{C}^{u \times v}$ stand for spaces of $u \times v$ real and complex matrices. 
$\nabla f(\cdot)$ and $\partial f(\cdot)$ denote the gradient and partial derivative of the function $f . \mathbf{I}_{\mathrm{u}}$ is the $\mathrm{u} \times \mathrm{u}$ unit diagonal matrix. The operators $|\cdot|$ and $\|\cdot\|_{2}$ denote the absolute value and the 2-norm of the Frobenius norm, respectively. The above-mentioned notations are shown in Table 1 as follows.

Table. 1 Notations

\begin{tabular}{|c|c|c|c|}
\hline Notation & Description & Notation & Description \\
\hline$(\cdot)^{T}$ & transpose & $\nabla f(\cdot)$ & gradient \\
\hline$(\cdot)^{H}$ & Hermitian transpose & $\partial f(\cdot)$ & partial derivative \\
\hline $\mathbf{E}[\cdot]$ & vector expectation operator & $\mathbf{I}_{\mathrm{u}}$ & absolute value \\
\hline $\mathbf{R}^{u \times v}$ & $u \times v$ real matrices & $|\cdot|$ & absolute value \\
\hline $\mathbf{C}^{u \times v}$ & $u \times v$ complex matrices & $\|\cdot\|_{2}$ & 2-norm of the Frobenius norm \\
\hline$E[\cdot]$ & scalar expectation operator & & \\
\hline
\end{tabular}

\section{Network Architecture and Complex ICA Model}

In this section, we mainly introduce the architecture of the WSNs and the complex ICA model. The architecture of the WSNs with BSS applied to solve the signal processing problems in the sink nodes is presented, described and analyzed in detail. And the block diagram of noise-free complex ICA in the sink nodes is also described and analyzed in detail.

\subsection{Network Architecture}

The architecture of WSNs with a separating module in the sink nodes is shown in Fig. 1, which consists of the sensor nodes, sink nodes and remote monitoring hosts (RMHs). The sink nodes receive, separate and transmit the signals of the sensor nodes to the RMHs. In this paper, the separating module in the sink nodes of the architecture employs the BSS method, and the ICA model is adopted to retrieve the signals from the sensor nodes. Obviously, the processing ability of the sink nodes is very important to the WSNs. The details are shown as follows.

Sensor nodes: Sensor nodes are used to sense different dynamic physical quantities in various scenarios, and convert the obtained sensor data into electrical signals [15]. Each sensor field is assumed to contain $N$ sensor nodes, and each sensor node is assumed to have a single antenna. Based on the property of wireless communications, the signals of sensor nodes are assumed to be statistically independent of each other. Due to the processing ability and power limit of the sensor nodes, sink nodes are required to gather the signals of sensor fields. 


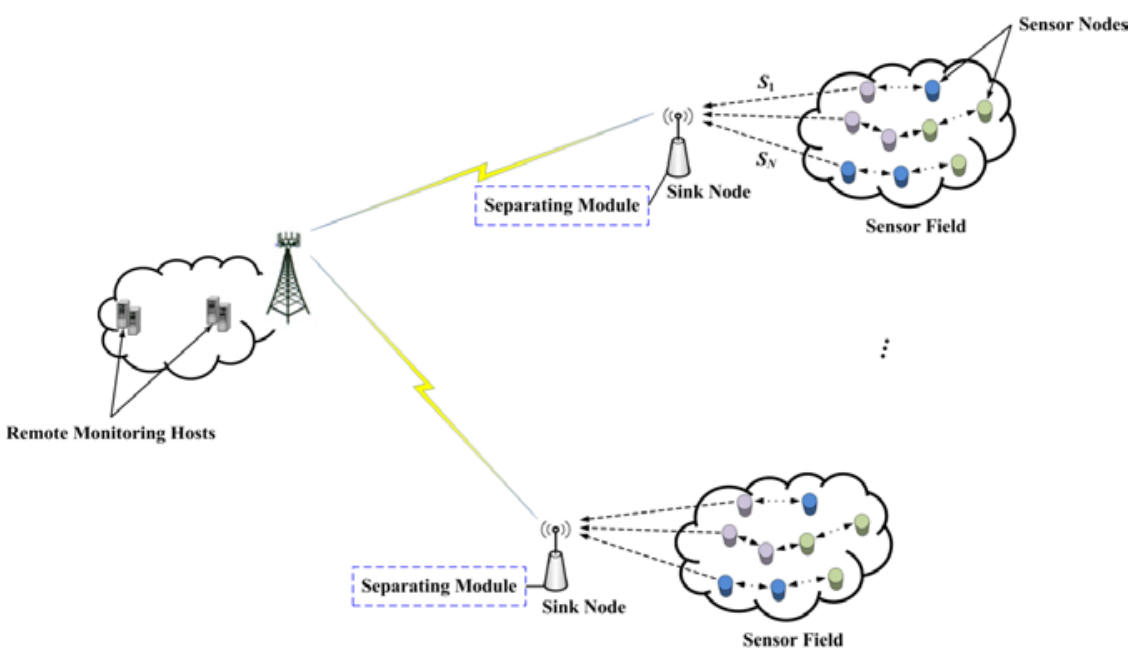

Fig. 1. The architecture of wireless sensor networks with a separating modulein the sink nodes.

Sink nodes: Sink nodes first receive the signals of the sensor nodes and noise sources from free space; then, they process the received noisy signals, ultimately transmitting the postprocessed signals to the RMHs. Each sink node is assumed to have $\mathrm{M}$ antennas and receive signals from its sensor field as shown in Fig. 1. As previously explained, the source signals of the sensor nodes are statistically independent, and the BSS method can be applied perfectly to separate the mixing received signals. Thus, there is little-to-no need of prior knowledge of the source signals of the sensor nodes, which is obviously advantageous in WSNs. Moreover, signal processing, such as signal separating and retrieving, in the sink nodes avoids final data centralization in the remote monitoring hosts; thus, a low data bit rate is achieved, and distributed signal processing algorithms can be applied.

Remote monitoring hosts: RMHs obtain the pre-processed information from the sink nodes and conduct further signal processing for the signals from the whole WSN.

Through the analysis above, it can be concluded that signal processing in the sink nodes is very important to the overall WSN. As a result, the block diagram of complex ICA for signal processing in the sink nodes is detailed in the following subsection.

\subsection{Complex ICA Model}

According to the fundamental principles of wireless communications, the block diagram of noise-free complex ICA in the sink nodes considered in this paper is shown in Fig. 2, which mainly includes unknown information of sources and the separating module. The source signals of the sensor nodes in the part of the unknown information of the sources, whether modulated or not, are going to be transmitted to the separating module, which represents the sink nodes in the WSNs in Fig. 1. In the separating module, each antenna receives $N$ mixing signals, which will be separated based on their differences in statistics (we used the independence of the source signals as the differences in statistics). Thus, the signals are obtained. The method in separating the signals successfully can be expressed for simplicity as followed. First, the statistics difference of the separated signals is calculated and weighted, if it equals that of the source signals, then the separation has been successfully achieved. Otherwise, the demixing matrix $\mathbf{W}$ has to be iteratively adjusted basing the contrast criteria by BSS algorithms until it has achieved a satisfactory separating performance. 


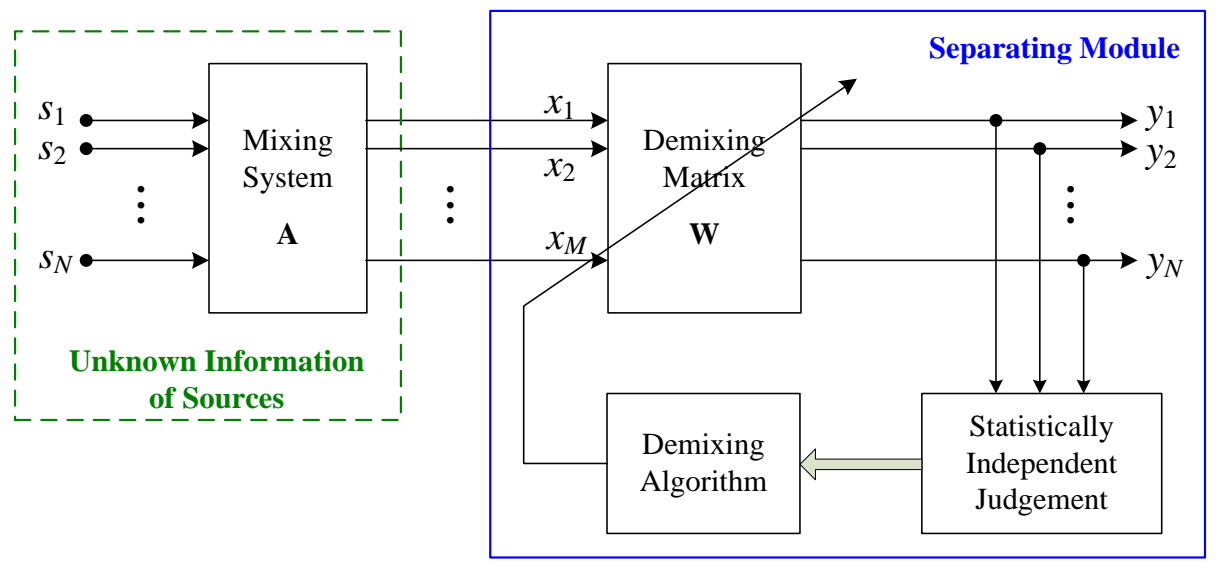

Fig. 2. Block diagram of complex ICA in sink nodes.

The mixing signals are given by

$$
\mathbf{x}(t)=\mathbf{A s}(t)
$$

where $\mathbf{s}(t)=\left[s_{1}(t), \ldots, s_{N}(t)\right]^{T}=\left[s_{1 r}(t)+i s_{1 i}(t), \ldots, s_{N r}(t)+i s_{N i}(t)\right]^{T}$ is the source signal vector of the sensor nodes, and the mixing matrix $\mathbf{A} \in \mathbf{R}^{M \times N}$, which denotes the property of wireless channel between the sensor nodes and the sink node, is composed of an M-row vectors, i.e., $\mathbf{A}=\left[\mathbf{a}_{1}, \mathbf{a}_{2}, \ldots, \mathbf{a}_{M}\right]^{T}$. The observed signal vector of the sink node is denoted by $\mathbf{x}(t)=\left[x_{1}(t), \ldots, x_{M}(t)\right]^{T}=\left[x_{1 r}(t)+i x_{1 i}(t), \ldots, x_{M r}(t)+i x_{M i}(t)\right]^{T}$. For complex random source signal vectors, $\operatorname{cov}(\mathbf{s}(t))=\mathbf{E}\left\{(\mathbf{s}(t)-\mathbf{E}\{\mathbf{s}(t)\})(\mathbf{s}(t)-\mathbf{E}\{\mathbf{s}(t)\})^{H}\right\}$ denotes the covariance matrix; and the pseudocovariance matrix can be written as $p \operatorname{cov}(\mathbf{s}(t))=\mathbf{E}\left\{(\mathbf{s}(t)-\mathbf{E}\{\mathbf{s}(t)\})(\mathbf{s}(t)-\mathbf{E}\{\mathbf{s}(t)\})^{T}\right\}$. These two quantities together define the second-order statistics of a complex random vector. The source vector is second-order circular or noncircular if $p \operatorname{cov}(\mathbf{s}(t))=0$ or $p \operatorname{cov}(\mathbf{s}(t)) \neq 0$. A stronger definition of circularity is based on the probability density function (PDF) of the complex random variable, which can be found in [42].

Similar to Eq. (1), we consider a separation operator, the output can be described as

$$
\mathbf{y}(\mathrm{t})=\mathbf{W}^{H} \mathbf{x}(\mathrm{t}),
$$

in which $\mathbf{W} \in \mathbf{R}^{M \times N}$ is the separating matrix containing $N$ column vectors, i.e., $\mathbf{W}=\left[\mathbf{w}_{1}, \mathbf{w}_{2}, \ldots, \mathbf{w}_{N}\right] \quad$ and the output signal vector $\mathbf{y}(\mathrm{t})=\left[y_{1}(\mathrm{t}), \ldots, y_{N}(\mathrm{t})\right]^{T}=\left[y_{1 r}(t)+i y_{1 i}(t), \ldots, y_{N r}(t)+i y_{N i}(t)\right]^{T}$ is the approximate estimation of the source signal vectors. Without loss of generality, we assume $M=N$ in this paper. 


\section{Contrast Criteria}

In this section, we introduce the reference signals and the contrast functions based on the reference signals at first. Then, we describe and prove in detail the local stability of the reference-based contrast criterion.

\subsection{Reference Signals}

As shown in [40], the above-mentioned reference signals are defined as

$$
\mathbf{z}(\mathrm{t})=\mathbf{V}^{H} \mathbf{x}(\mathrm{t})
$$

where $\mathbf{z}(\mathrm{t})=\left[z_{1}(\mathrm{t}), \ldots, z_{N}(\mathrm{t})\right]^{T}=\left[z_{1 r}(t)+i z_{1 i}(t), \ldots, z_{N r}(t)+i z_{N i}(t)\right]^{T}$ is the reference signal vector. $\mathbf{z}(\mathrm{t})=\left[z_{1}(\mathrm{t}), \ldots, z_{N}(\mathrm{t})\right]^{T}=\left[z_{1 r}(t)+i z_{1 i}(t), \ldots, z_{N r}(t)+i z_{N i}(t)\right]^{T}$ are in a similar form as $\mathbf{y}(\mathrm{t})=\mathbf{W}^{H} \mathbf{x}(\mathrm{t})$. In addition, it should be noted that the initialization of $\mathbf{V}$ has an important influence on the performance of the algorithms. Moreover, the reference signals are artificially introduced in the algorithms for the purpose of facilitating the maximization of the contrast functions. Because no confusion is possible, and for simplicity, in the following sections, we drop the time index of these vectors, which are denoted by $\mathbf{s}, \mathbf{x}, \mathbf{y}$ and $\mathbf{z}$, respectively.

\subsection{Contrast Functions}

First of all, we use the following definitions for random vectors throughout this paper.

$$
\begin{aligned}
& \mathbf{u}=\left[u_{1}, u_{2}, \ldots, u_{N}\right]^{T} \in \mathbf{C}^{N}, \\
& \overline{\mathbf{u}}=\left[u_{1}, u_{1}^{*}, \ldots, u_{N}, u_{N}^{*}\right]^{T} \in \mathbf{C}^{2 N}, \\
& \overline{\overline{\mathbf{u}}}=\left[u_{1}^{R}, u_{1}^{I}, \ldots, u_{N}^{R}, u_{N}^{I}\right]^{T} \in \mathbf{C}^{2 N},
\end{aligned}
$$

where $u_{i}=u_{i}^{R}+j u_{i}^{I}, i=1,2, \ldots, N$ are the elements of $\mathbf{u}$.

Second, as defined in [42], the gradient and Hessian forms for a complex analytic function $f$ are expressed as

$$
\begin{aligned}
& \nabla_{\mathbf{u}} f\left(\mathbf{u}_{0}\right)=\left.\frac{\partial f(\mathbf{u})}{\partial \overline{\mathbf{u}}}\right|_{\mathbf{u}=\mathbf{u}_{0}}, \\
& \nabla_{\mathbf{u}}^{2} f\left(\mathbf{u}_{0}\right)=\left.\frac{\partial^{2} f(\mathbf{u})}{\partial \overline{\mathbf{u}}^{*} \partial \overline{\mathbf{u}}^{T}}\right|_{\mathbf{u}=\mathbf{u}_{0}},
\end{aligned}
$$

where

$$
\begin{aligned}
& \frac{\partial f}{\partial u}=\frac{1}{2}\left(\frac{\partial f}{\partial u^{R}}-j \frac{\partial f}{\partial u^{I}}\right), \\
& \frac{\partial f}{\partial u^{*}}=\frac{1}{2}\left(\frac{\partial f}{\partial u^{R}}+j \frac{\partial f}{\partial u^{I}}\right) .
\end{aligned}
$$

Finally, it was shown that for $p(u)=f_{1}\left(u^{*} x\right) f_{2}^{*}\left(u^{*} x\right)$, where $f_{1}$ and $f_{2}$ are analytic functions, we have [42] 


$$
\begin{aligned}
& \frac{\partial p(u)}{\partial u}=x^{*} f_{1}\left(u^{*} x\right) f_{2}^{\prime^{*}}\left(u^{*} x\right), \\
& \frac{\partial p(u)}{\partial u^{*}}=x f_{1}^{\prime}\left(u^{*} x\right) f_{2}^{*}\left(u^{*} x\right),
\end{aligned}
$$

where $f^{\prime}(u)=\frac{d f(u)}{d u}$.

Then, the following contrast functions is given by

$$
\begin{aligned}
& J(\mathbf{w})=\mathbf{E}\left\{\left|G\left(\mathbf{w}^{H} \mathbf{x}\right)\right|^{2}\right\}, \\
& I(\mathbf{w}, \mathbf{v})=\mathbf{E}\left\{G\left(\mathbf{w}^{H} \mathbf{x}\right) G\left(\mathbf{v}^{H} \mathbf{x}\right)^{*}\right\},
\end{aligned}
$$

where $J$ is the negentropy proposed by Novey [41] and $I$ is the reference-based contrast function, which has been proposed by introducing reference signals into $J . \mathbf{w}$ and $\mathbf{v}$ are the column vectors of $\mathbf{W}$ and $\mathbf{V}$, respectively. The nonlinear functions $G$ are chosen as follows [39]:

$$
\begin{aligned}
& G_{1}(y)=a \sinh (y)=\ln \left(y+\sqrt{y^{2}+1}\right), \\
& g_{1}(y)=\frac{\sqrt{y^{2}+1}+y}{y \sqrt{y^{2}+1}+y^{2}+1}, \\
& g_{1}^{\prime}(y)=-\frac{2 y^{3}+2 y^{2} \sqrt{y^{2}+1}+y}{\left(y \sqrt{y^{2}+1}+y^{2}+1\right)^{2} \sqrt{y^{2}+1}}, \\
& G_{2}(y)=\cosh (y)=\frac{e^{y}+e^{-y}}{2}, \\
& g_{2}(y)=\frac{e^{y}-e^{-y}}{2}, \\
& g_{2}^{\prime}(y)=\frac{e^{y}+e^{-y}}{2}, \\
& G_{3}(y)=y^{1.25}, \\
& g_{3}(y)=1.25 y^{0.25}, \\
& g_{3}^{\prime}(y)=0.3125 y^{-0.75},
\end{aligned}
$$

where $G: \mathbf{C} \rightarrow \mathbf{C}$. $g_{i}$ and $g_{i}^{\prime}$ are the derivatives of $G_{i}$ and $g_{i}$ respectively. The stability of the contrast function in Eq. (9) is proved in the next subsection.

\subsection{Stability Analysis}

In this subsection, we investigate the stability of $I(\mathbf{w}, \mathbf{v})$ by using a second-order approximation of $I(\mathbf{w}, \mathbf{v})$ around the stable points. The approximation performs well when the higher order terms of a Taylor series are negligible and the Hessian exists at the stable point [38]. The local consistency of $I(\mathbf{w}, \mathbf{v})$ is investigated under the constraint $\|\mathbf{w}\|^{2}=1$. We make the orthogonal change in coordinates $\mathbf{p}=\mathbf{w}^{H} \mathbf{A}$ and analyze the stability of such $\mathbf{p}$. 
Without loss of generality, we assume that the optimal solution is given by $\mathbf{p}_{1}=\left[p_{1}, p_{2}, \ldots, p_{N}\right]^{T}=\left[e^{j \theta}, 0, \ldots, 0\right]^{T}$ in this paper.

Under the assumptions on sources, the observed signal variables $x_{n}, n=1,2, \ldots, N$ in $\mathbf{x}$ are prewhitened using $\mathbf{E}\left\{\mathbf{x x}^{H}\right\}=\mathbf{I}$. As shown in the Appendix, the extrema for noncircular sources in the complex plane of $p_{1}=e^{j \theta}$ is given by

$$
\theta=\frac{1}{2}\left(\frac{2 \mathbf{E}\left\{\left(s^{R}\right)^{3} s^{I}+\left(s^{I}\right)^{3} s^{R}\right\}}{\mathbf{E}\left\{\left(s^{R}\right)^{4}-\left(s^{I}\right)^{4}\right\}}\right)+n \pi,
$$

and we obtain the condition along dimension $p_{1}$ for a minimum (resp. maximum) as

$$
\begin{aligned}
-2 \mathbf{E}\left\{G^{*}\left(e^{-j \theta} s_{1}\right) g\left(e^{-j \theta} s_{1}\right) s_{1}\right\}> & \mathbf{E}\left\{s_{i}^{2} G^{*}\left(e^{j \theta} s_{1}\right) g^{\prime}\left(e^{j \theta} s_{1}\right) e^{-j 2 \theta}\right. \\
& \left.+\left(s_{i}^{2} G^{*}\left(e^{j \theta} s_{1}\right) g^{\prime}\left(e^{j \theta} s_{1}\right)\right)^{*} e^{-j 2 \theta}\right\},(\text { resp. }<0) .
\end{aligned}
$$

Then, we extend the stability conditions with respect to $p_{1}$ to the other dimensions i.e., $p_{2}, \ldots, p_{N}$ by using the Hessian analysis. For a given source $s_{i}, i=2, \ldots, N$, a local minimum (resp. maximum) is achieved when

$$
-\mathbf{E}\left\{G^{*}\left(e^{-j \theta} s_{1}\right) g\left(e^{-j \theta} s_{1}\right) s_{1}\right\} e^{-j \theta} \pm\left|s_{i}^{2} G^{*}\left(e^{j \theta} s_{1}\right) g^{\prime}\left(e^{j \theta} s_{1}\right)\right|>0,(\text { resp. }<0) .
$$

Note that in Eqs. (14) and (15), source $i=1$ is chosen as an example to show the source-to-source stability dependence and must be true for all source combinations, i.e., estimating source $i=1$ with given sources $i=2,3, \ldots, N$. The stability proof in detail can be found in the Appendix.

\section{Derivation of RSNT-cFastICA Algorithm}

In this section, we mainly show the process to derive the RSNT-cFastICA algorithm and analyze its stability condition in detail based on the above section 3 .

Similar to [39] and [41], the Lagrangian function under the constraint $\|\mathbf{w}\|=1$ is constructed as

$$
L(\mathbf{w}, \lambda)=I(\mathbf{w}, \mathbf{v})+\lambda\left(\mathbf{w} \mathbf{w}^{H}-1\right),
$$

where $\lambda$ is the real-valued Lagrange multiplier and $\|\mathbf{v}\|=1$ because $\mathbf{v}$ updates following $\mathbf{w}$. As shown in [39], we use the complex Newton update defined in [42].

Then, the Newton iteration can be formulated as

$$
\begin{aligned}
\overline{\mathbf{w}}_{n+1} & =\overline{\mathbf{w}}_{n}-\left.\left(\left.\frac{\partial^{2} L(\overline{\mathbf{w}}, \lambda)}{\partial \overline{\mathbf{w}} \mathbf{w}^{*} \partial \overline{\mathbf{w}} \mathbf{w}^{T}}\right|_{\mathbf{w}=\mathbf{w}_{n}}\right)^{-1} \frac{\partial L(\overline{\mathbf{w}}, \lambda)}{\partial \overline{\mathbf{w}}^{*}}\right|_{\mathbf{w}=\mathbf{w}_{n}} \\
& =\overline{\mathbf{w}}_{n}-\left.\left(\left.\left(\frac{\partial^{2} I(\overline{\mathbf{w}}, \overline{\mathbf{v}})}{\partial \overline{\mathbf{w}}^{*} \partial \overline{\mathbf{w}}^{T}}+\lambda \overline{\mathbf{I}}\right)\right|_{\mathbf{w}=\mathbf{w}_{n}}\right)^{-1}\left(\frac{\partial I(\overline{\mathbf{w}}, \overline{\mathbf{v}})}{\partial \overline{\mathbf{w}}^{*}}+\lambda \overline{\mathbf{w}}\right)\right|_{\mathbf{w}=\mathbf{w}_{n}} \\
\overline{\mathbf{w}}_{n+1} & =\frac{\overline{\mathbf{w}}_{n+1}}{\left\|\overline{\mathbf{w}}_{n+1}\right\|},
\end{aligned}
$$


where Eqs. (4)-(7) are used.

Now, by multiplying both sides of Eq. (17) with complex Hessian $\frac{\partial^{2} I(\overline{\mathbf{w}}, \overline{\mathbf{v}})}{\partial \overline{\mathbf{w}}^{*} \partial \overline{\mathbf{w}}^{T}}+\lambda \overline{\mathbf{I}}$, we can further simplify Eq. (17) as

$$
\begin{aligned}
& \overline{\mathbf{w}}_{n+1}=\left(\left.\left(\frac{\partial^{2} I(\overline{\mathbf{w}}, \overline{\mathbf{v}})}{\partial \overline{\mathbf{w}}^{*} \partial \overline{\mathbf{w}}^{T}}+\lambda \overline{\mathbf{I}}\right)\right|_{\mathbf{w}=\mathbf{w}_{n}}\right) \overline{\mathbf{w}}_{n}-\left.\left(\frac{\partial I(\overline{\mathbf{w}}, \overline{\mathbf{v}})}{\partial \overline{\mathbf{w}}^{*}}+\lambda \overline{\mathbf{v}}\right)\right|_{\mathbf{w}=\mathbf{w}_{n}}, \\
& \overline{\mathbf{w}}_{n+1}=\frac{\overline{\mathbf{w}}_{n+1}}{\left\|\overline{\mathbf{w}}_{n+1}\right\|},
\end{aligned}
$$

where $\frac{\partial I(\overline{\mathbf{w}}, \overline{\mathbf{v}})}{\partial \overline{\mathbf{w}}^{*}}, \frac{\partial^{2} I(\overline{\mathbf{w}}, \overline{\mathbf{v}})}{\partial \overline{\mathbf{w}}^{*} \partial \overline{\mathbf{w}}^{T}}$ are defined as the following Eq. (19) and Eq. (20), respectively.

$$
\begin{aligned}
& \frac{\partial I(\overline{\mathbf{w}}, \overline{\mathbf{v}})}{\partial \overline{\mathbf{w}}^{*}}=\left(\begin{array}{c}
\frac{\partial I(\overline{\mathbf{w}}, \overline{\mathbf{v}})}{\partial w_{1}{ }^{*}} \\
\frac{\partial I(\overline{\mathbf{w}}, \overline{\mathbf{v}})}{\partial w_{1}} \\
\vdots \\
\frac{\partial I(\overline{\mathbf{w}}, \overline{\mathbf{v}})}{\partial w_{N}{ }^{*}} \\
\frac{\partial I(\overline{\mathbf{w}}, \overline{\mathbf{v}})}{\partial w_{N}}
\end{array}\right)=\mathbf{E}\left(\begin{array}{c}
x_{1} g\left(\overline{\mathbf{w}}^{H} \overline{\mathbf{x}}\right) G\left(\overline{\mathbf{v}}^{H} \overline{\mathbf{x}}\right)^{*} \\
0 \\
\vdots \\
x_{N} g\left(\overline{\mathbf{w}}^{H} \overline{\mathbf{x}}\right) G\left(\overline{\mathbf{v}}^{H} \overline{\mathbf{x}}\right)^{*} \\
0
\end{array}\right),
\end{aligned}
$$

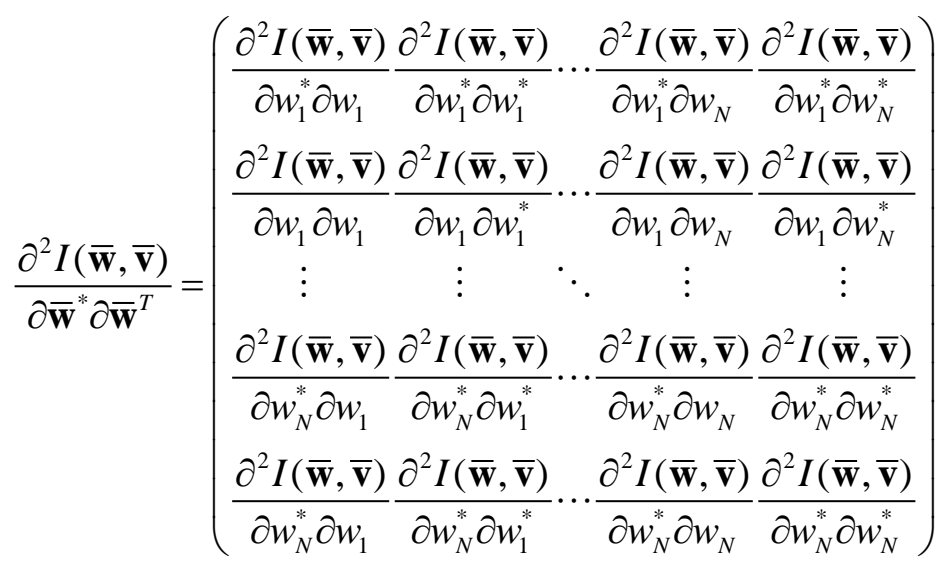

$$
\begin{aligned}
& =\left(\begin{array}{ccccc}
0 & x_{1} x_{1} \alpha & \cdots & 0 & x_{1} x_{N} \alpha \\
0 & 0 & \cdots & 0 & 0 \\
\vdots & \vdots & \ddots & \vdots & \vdots \\
0 & x_{N} x_{1} \alpha & \cdots & 0 & x_{N} x_{N} \alpha \\
0 & 0 & \cdots & 0 & 0
\end{array}\right) \text {, }
\end{aligned}
$$

$\alpha=g^{\prime}\left(\overline{\mathbf{w}}^{H} \overline{\mathbf{x}}\right) G\left(\overline{\mathbf{v}}^{H} \overline{\mathbf{x}}\right)^{*}, g$ and $g^{\prime}$ are the derivatives of $G$ and $g$, respectively. 
Expanding and simplifying $\frac{\partial I(\overline{\mathbf{w}}, \overline{\mathbf{v}})}{\partial \overline{\mathbf{w}}^{*}}$ and $\frac{\partial^{2} I(\overline{\mathbf{w}}, \overline{\mathbf{v}})}{\partial \overline{\mathbf{w}}^{*} \partial \overline{\mathbf{w}}^{T}} \overline{\mathbf{w}}$ by retaining the even-numbered rows, we have

$$
\begin{aligned}
& \frac{\partial I(\mathbf{w}, \mathbf{v})}{\partial \mathbf{w}^{*}}=\mathbf{E}\left(\begin{array}{c}
x_{1} g\left(\mathbf{w}^{H} \mathbf{x}\right) G\left(\mathbf{v}^{H} \mathbf{x}\right)^{*} \\
x_{2} g\left(\mathbf{w}^{H} \mathbf{x}\right) G\left(\mathbf{v}^{H} \mathbf{x}\right)^{*} \\
\vdots \\
x_{N} g\left(\mathbf{w}^{H} \mathbf{x}\right) G\left(\mathbf{v}^{H} \mathbf{x}\right)^{*}
\end{array}\right)=\mathbf{E}\left(\mathbf{x} g\left(\mathbf{w}^{H} \mathbf{x}\right) G\left(\mathbf{v}^{H} \mathbf{x}\right)^{*}\right), \\
& \begin{aligned}
\frac{\partial^{2} I(\mathbf{w}, \mathbf{v})}{\partial \mathbf{w}^{*} \partial \mathbf{w}^{T}} \mathbf{w}^{*} & =\mathbf{E}\left(\begin{array}{cccc}
x_{1} x_{1} \alpha & x_{1} x_{2} \alpha & \cdots & x_{1} x_{N} \alpha \\
x_{2} x_{1} \alpha & x_{2} x_{2} \alpha & \cdots & x_{2} x_{N} \alpha \\
\vdots & \vdots & \ddots & \vdots \\
x_{N} x_{1} \alpha & x_{N} x_{2} \alpha & \cdots & x_{N} x_{N} \alpha
\end{array}\right) \mathbf{w}^{*} \\
& =\mathbf{E}\left\{g^{\prime}\left(\mathbf{w}^{H} \mathbf{x}\right) G\left(\mathbf{v}^{H} \mathbf{x}\right)^{*} \mathbf{x} \mathbf{x}^{T}\right\} \mathbf{w}^{*} \\
& \approx \mathbf{E}\left\{g^{\prime}\left(\mathbf{w}^{H} \mathbf{x}\right) G\left(\mathbf{v}^{H} \mathbf{x}\right)^{*}\right\} \mathbf{E}\left\{\mathbf{x x}^{T}\right\} \mathbf{w}^{*} .
\end{aligned}
\end{aligned}
$$

Substituting Eqs.(21) and (22) into Eq.(17), we can reformulate Eq. (17) as follows:

$$
\begin{aligned}
& \mathbf{w}_{n+1}=-\mathbf{E}\left(\mathbf{x} g\left(\mathbf{w}_{n}^{H} \mathbf{x}\right) G\left(\mathbf{v}_{n}^{H} \mathbf{x}\right)^{*}\right)+\mathbf{E}\left\{g^{\prime}\left(\mathbf{w}_{n}^{H} \mathbf{x}\right) G\left(\mathbf{v}_{n}^{H} \mathbf{x}\right)^{*}\right\} \mathbf{E}\left\{\mathbf{x} \mathbf{x}^{T}\right\} \mathbf{w}_{n}^{*}, \\
& \mathbf{w}_{n+1}=\frac{\mathbf{w}_{n+1}}{\left\|\mathbf{w}_{n+1}\right\|} .
\end{aligned}
$$

\section{Simulation Results and Analysis}

In this section, we mainly illustrate and validate the separability, effectiveness and complexity of the RSNT-cFastICA algorithm based on anlytical and computer simulation results.

\subsection{Separability Analysis}

In this section, we explore the validity of the RSNT-cFastICA algorithm by choosing three noncircular 4-QAM signals as sources. Noncircular sources are generated with different values of real-to-imaginary asymmetry defined by

$$
\eta=\frac{\mathbf{E}\left\{\left(s^{R}\right)^{2}\right\}}{\mathbf{E}\left\{\left(s^{I}\right)^{2}\right\}} .
$$

Then by providing a random phase shift, real-to-imaginary correlation values are obtained [40]. Here, source signals from sink nodes $N=3$, real-to-imaginary asymmetry values $\eta=10$, and the number of samples $T=5000$ are considered. The sources are shown in Fig. 3. After mixing the sources using a random matrix, we obtain the mixed signals shown in Fig. 4. Using RSNT-cFastICA algorithm with the nonlinear function $G_{2}$ in Eq.(11), we obtain the separations in Fig. 5. It can be clearly observed that the recovered signals in Fig. $\mathbf{5}$ are the estimations of sources in Fig. 3 up to permutation and scaling ambiguities. These inherent ambiguities are common to BSS problems and are insignificant in most practical applications, because most of the useful information of the signals is preserved in the waveforms of the signals. This problem is discussed in detail in the following works. 

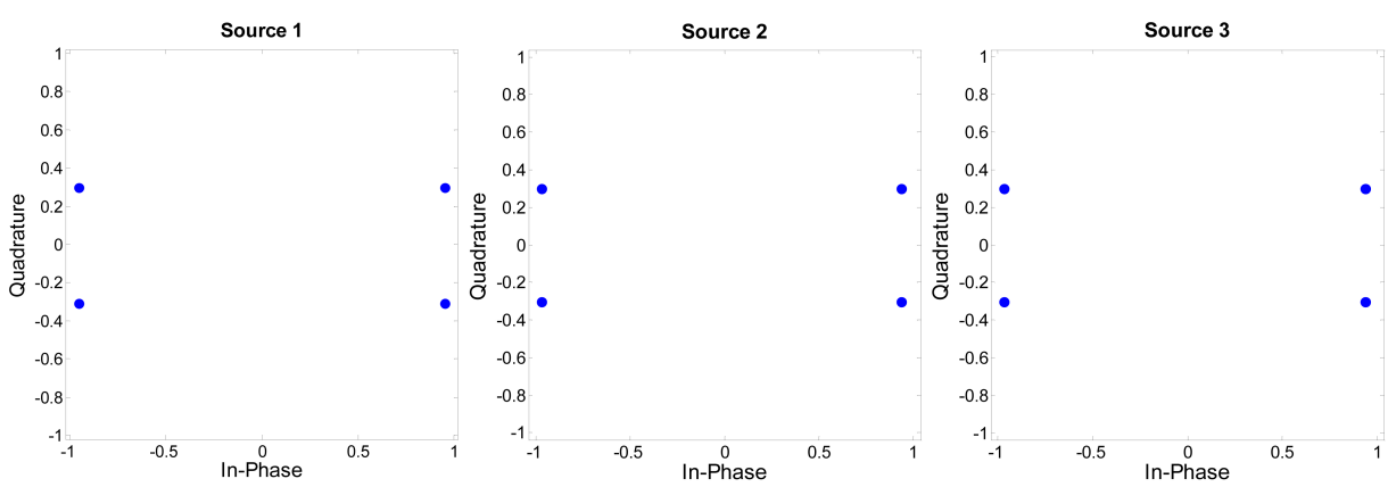

Fig. 3. Three noncircular source signals.
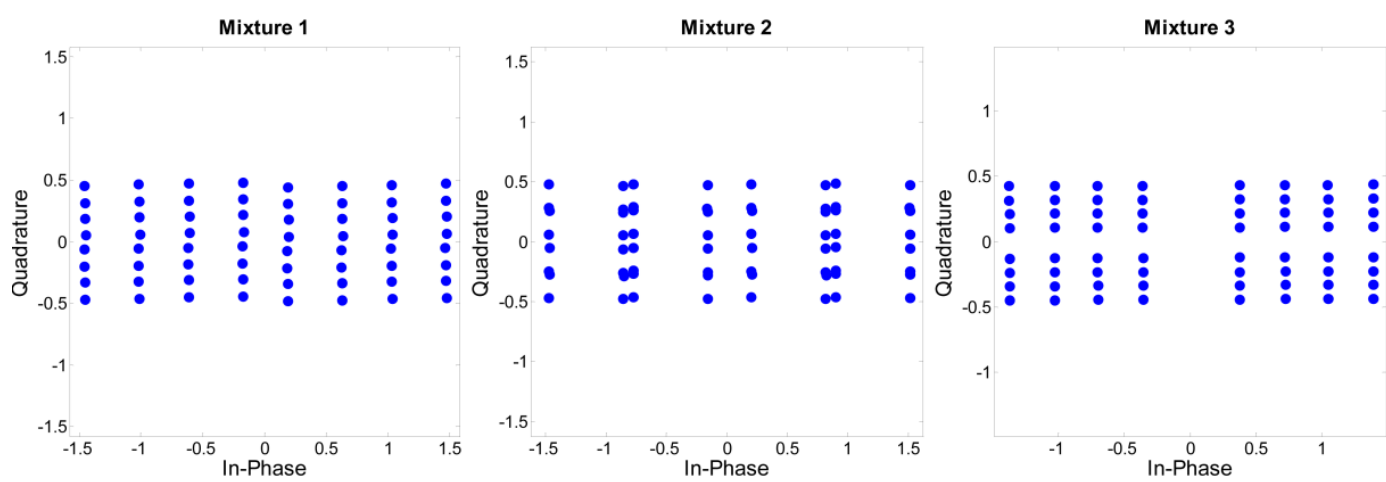

Fig. 4. Three mixing signals
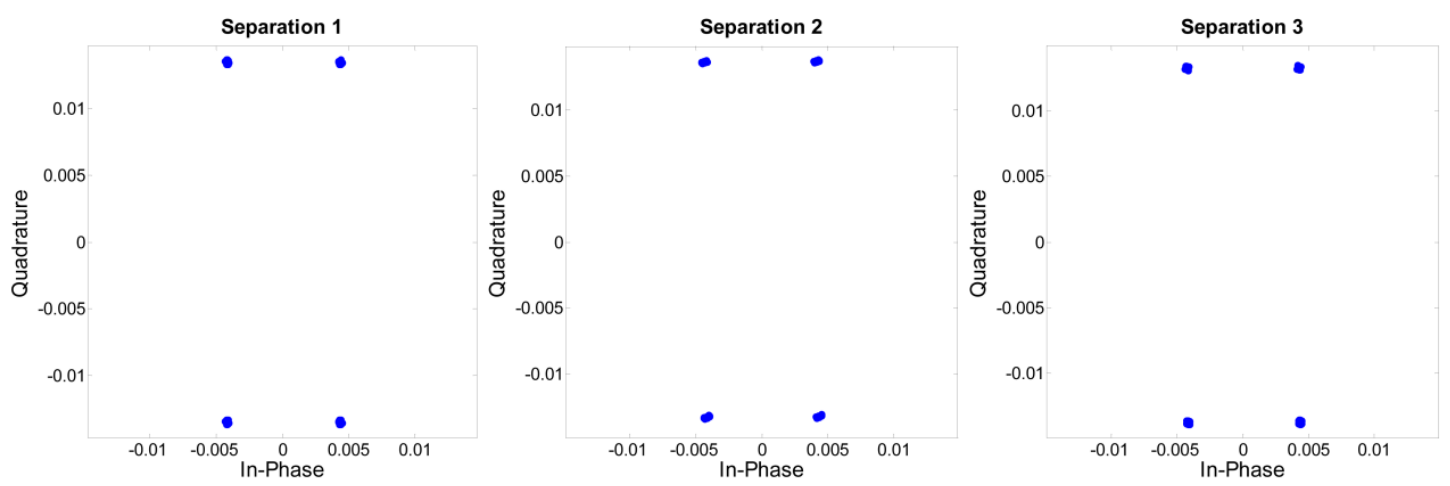

Fig. 5. Three separated signals.

\subsection{Effectiveness Analysis}

In this subsection, the performance of the proposed RSNT-cFastICA algorithm is verified using complex-valued sub-Gaussian and super-Gaussian sources with both circular and noncircular distributions. The performance of the RSNT-cFastICA algorithm using analytic functions $G_{1}, G_{2}$, and $G_{3}$ is compared with JADE [43], c-FastICA [44] using the contrast function $G(y)=\log (1+y)$ and quasi-Newton algorithm using Eq. (10) to Eq. (12) in [39]. The definitions of JADE, C-fastICA, asinh, cosh, and $z^{1.25}$ are used for the latter algorithms and asinh $+R$, $\cosh +R$, and $z^{1.25}+R$ are used for RSNT-cFastICA in Fig. 6, respectively. 


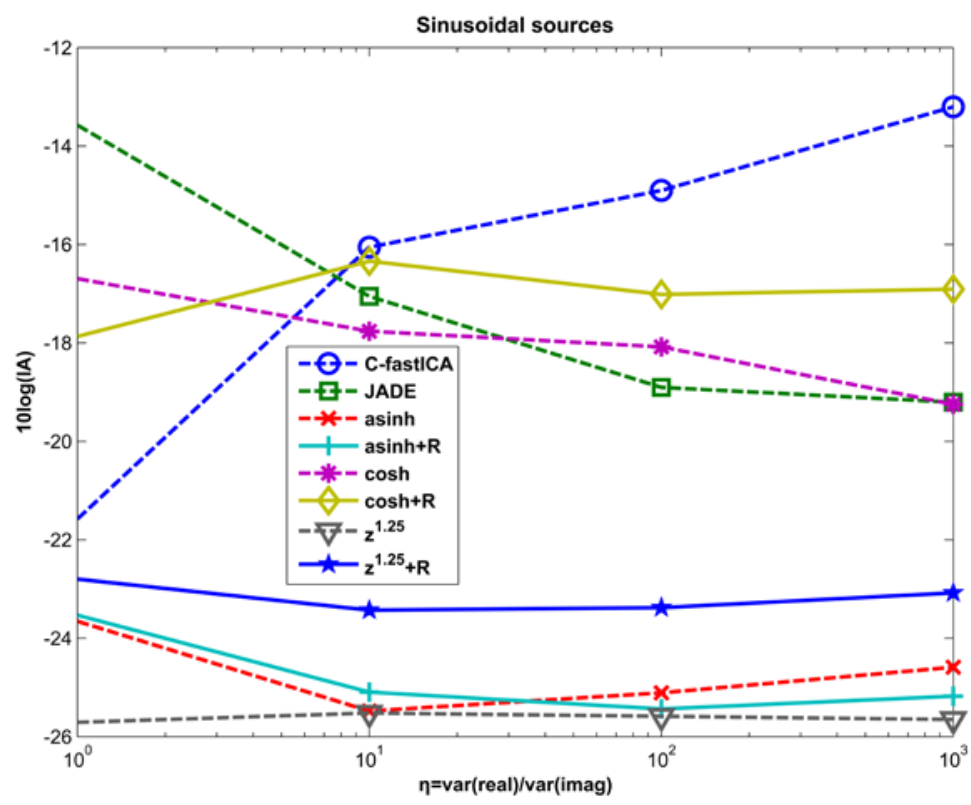

Fig. 6. Average IA as a function of the asymmetry of the real-to-imaginary parts $\eta$ of eight complex sinusoidal sources.

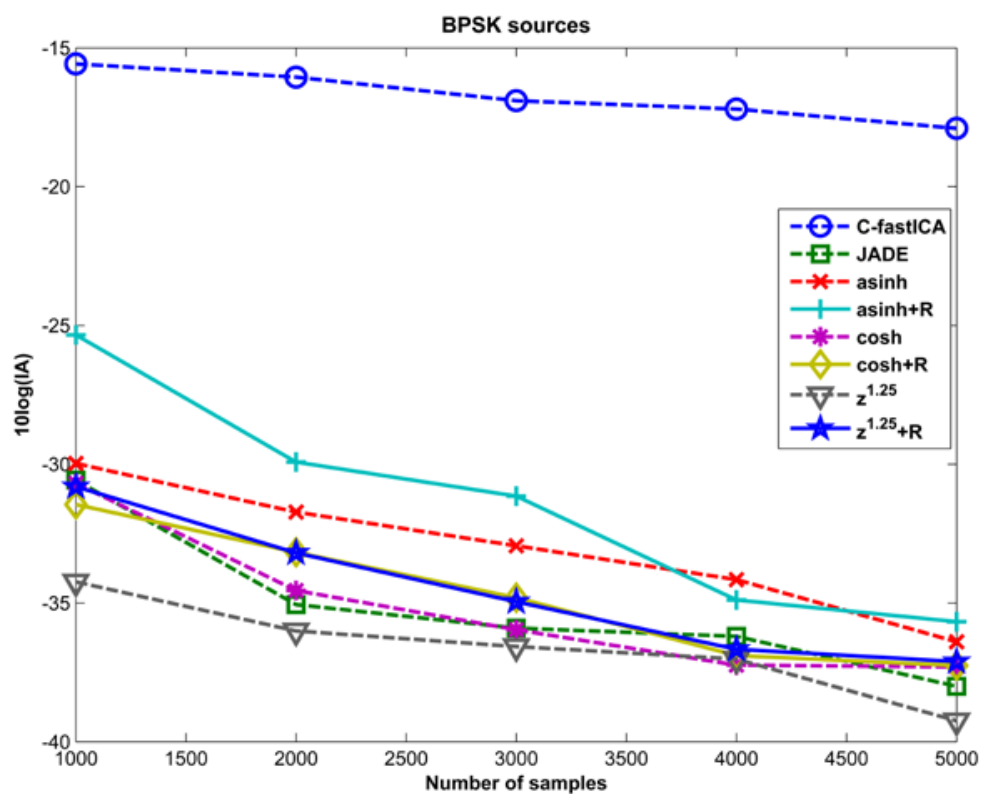

Fig. 7. Average IA as a function of data length for a mixture of eight complex BPSK sources.

The normalized Amari index in [39] is used for the performance measurement.

$$
\mathbf{I A}=\frac{1}{2 N(N-1)} \sum_{m}\left(\sum_{n} \frac{\left|p_{m n}\right|^{2}}{\left(\max \left|\mathbf{p}_{m}\right|\right)^{2}}-1\right)+\frac{1}{2 N} \sum_{n}\left(\sum_{m} \frac{\left|p_{m n}\right|^{2}}{\left(\max \left|\mathbf{p}_{n}\right|\right)^{2}}-1\right),
$$

where $\mathbf{P}=$ WQA and $\mathbf{Q}$ is the whitening matrix. The lower the value is, the better the separation performance becomes, with $10 \log \mathbf{I A}>-10 \mathrm{~dB}$ indicating that the algorithm is not 
performing adequately. With respect to the computational speed, the execution time is used as the measurement criterion. The computer that we utilize is equipped Intel (R) $\operatorname{Core}^{T M} 2$ Duo CPU, E8400 @ 3.0 GHz, 2.99 GHz, and 3.00 GB of RAM. All simulations are averaged over 100 runs with exactly the same matrices and sources realized on each run, and the simulation results are illustrated in Fig. 6 to Fig. 8.

In Fig. 6, eight complex sinusoidal signals from $N=8$ sensor nodes are considered as sources. The definition of noncircular sources with different values of real-to-imaginary asymmetry $\eta$ can be found in Eq. (24). The other corresponding parameters are as follows: $T=500$ and $\eta=10^{0}, 10^{1}, 10^{2}, 10^{3}$. In Fig. 7 and Fig. 8, we choose eight BPSK signals as sources. The corresponding parameters are as follows: $N=8$, and $T=1000,2000,3000,4000,5000$.

As observed from Fig. 6, for sub-Gaussian sources, the performance of the RSNT-cFastICA algorithm is close to that of the quasi-Newton algorithm, especially when $\eta$ is increasing, whereas C-fastICA performs worse. In other words, the proposed approach is approximately as efficient as the original approach in terms of separation quality, especially for noncircular sources.

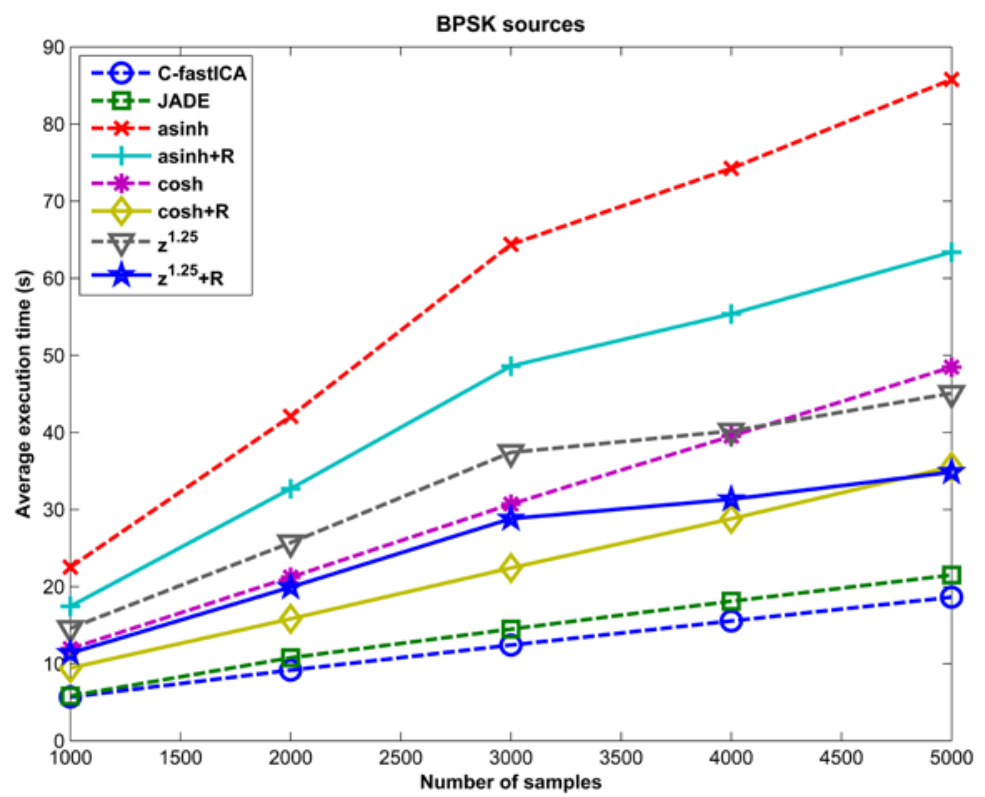

Fig. 8. Average execution time for retrieving eight complex BPSK sources.

It can be observed clearly from Fig. 6 that all the algorithms perform equally well for BPSK sources except for C-fastICA. Their IA values of them are less than $-25 \mathrm{~dB}$, especially when the number of samples increases, whereas the IA of C-fastICA is approxiately $-17 \mathrm{~dB}$. It can be concluded that RSNT-cFastICA algorithm using asinh, cosh, and $z^{1.25}$ can provide an equal performance to Novey's quasi-Newton algorithm.

However, from Fig. 8, it can be clearly observed that the execution time of the RSNT-cFastICA method using $G 1, G 2$, and $G 3$ is less than that of Novey's quasi-Newton algorithm, which is especially apparent with the increasing sample size. Combining with Fig. 7, we can draw the conclusion that the proposed algorithm can provide an equal performance to the original quasi-Newton algorithm, especially for the sub-Gaussian sources that are noncircular. Moreover, the algorithm is substantially more efficient than the latter in terms of 
computation speed with large numbers of samples, which can improve the power consumption effeciency of the sink nodes.

\subsection{Complexity Analysis}

As shown in Eq.(23), the core iteration step of our algorithm is

$$
\mathbf{w}_{n+1}=-\mathbf{E}\left(\mathbf{x} g\left(\mathbf{w}_{n}^{H} \mathbf{x}\right) G\left(\mathbf{v}_{n}^{H} \mathbf{x}\right)^{*}\right)+\mathbf{E}\left\{g^{\prime}\left(\mathbf{w}_{n}^{H} \mathbf{x}\right) G\left(\mathbf{v}_{n}^{H} \mathbf{x}\right)^{*}\right\} \mathbf{E}\left\{\mathbf{x x}^{T}\right\} \mathbf{w}_{n}^{*} .
$$

And the corresponding step of Novey's quasi-Newton algorithm is

$$
\begin{aligned}
\mathbf{w}_{n+1}= & -\mathbf{E}\left\{\mathbf{x} G^{*}\left(\mathbf{w}_{n}^{H} \mathbf{x}\right) g\left(\mathbf{w}_{n}^{H} \mathbf{x}\right)\right\}+\mathbf{E}\left\{g^{*}\left(\mathbf{w}_{n}^{H} \mathbf{x}\right) g\left(\mathbf{w}_{n}^{H} \mathbf{x}\right)\right\} \mathbf{w}_{n} \\
& +\mathbf{E}\left\{\mathbf{x} \mathbf{x}^{T}\right\} \mathbf{E}\left\{G^{*}\left(\mathbf{w}_{n}^{H} \mathbf{x}\right) g^{\prime}\left(\mathbf{w}_{n}^{H} \mathbf{x}\right)\right\} \mathbf{w}_{n}^{*} .
\end{aligned}
$$

It can be observed that the difference term between Eqs.(26) and (27) is $\mathbf{E}\left\{g^{*}\left(\mathbf{w}_{n}^{H} \mathbf{x}\right) g\left(\mathbf{w}_{n}^{H} \mathbf{x}\right)\right\} \mathbf{w}_{n}$, which is an increasing function of the number of sources, sample size, and the iteration time. When the iteration time and source size are fixed, the extra term $\mathbf{E}\left\{g^{*}\left(\mathbf{w}_{n}^{H} \mathbf{x}\right) g\left(\mathbf{w}_{n}^{H} \mathbf{x}\right)\right\} \mathbf{w}_{n}$ increases with the increasing of the number of samples. Therefore, the computational cost of RSNT-cFastICA algorithm is less than that of Novey's quasi-Newton algorithm, especially when the number of sample increases. Together with the simulations above, it can be observed that, for RSNT-cFastICA, decreasing the computational cost is of equal effectiveness in obtaining improving of the power consumption effeciency of the sink nodes, which is obviously significant for WSNs and WCNs.

\section{Conclusion}

In this paper, the architecture of WSNs with BSS applied to solve the signal retrieving problems in the sink nodes was first presented, and each component of the architecture was analyzed in detail. Then by introducing the reference-based scheme to negentropy, the RSNT-cFastICA method was proposed to solve the complex-valued noncircular sources of the sink nodes in WSNs. Theoretical analysis showed that RSNT-cFastICA not only contained an equal separation quality to Novey's quasi-Newton algorithm, but also presented its own advantage in that its computational speed was much higher than the latter especially when the number of samples was large, which was also validated by simulations. By comparing with JADE, c-FastICA and quasi-Newton algorithm under different conditions through simulations, the effectiveness of the RSNT-cFastICA method was proved. Simultaneously, decreasing of the computational cost of the proposed RSNT-cFastICA method leads to the improving of the power consumption effeciency in the sink nodes, which will obviously be significant when applied to WSNs and WCNs. Future work will include applying the underdetermined BSS method to WSNs to further improve the power consumption efficiency of the network.

\section{Appendix}

To investigate the local stability of the new contrast function $I$, we construct a Lagrangian function under the constraint $\mathbf{E}\left\{\left|\mathbf{w}^{H} \mathbf{x}\right|^{2}\right\}=\|\mathbf{w}\|^{2}=1$ as

$$
L(\mathbf{p}, \lambda)=I(\mathbf{p}, \mathbf{q})+\lambda(h(\mathbf{p}))=\mathbf{E}\left\{G(\mathbf{p s}) G(\mathbf{q} \mathbf{s})^{*}\right\}+\lambda\left(\|\mathbf{p}\|^{2}-1\right),
$$

at the optimal solution $\mathbf{p}_{1}=\left[e^{j \theta}, 0, \ldots, 0\right]$, where $\lambda$ is the real-valued Lagrange multiplier and 
$\|\mathbf{q}\|^{2}=1$ for $\mathbf{q}$ updates following $\mathbf{p}$. As shown in [42], the second-order necessary and sufficient conditions for a local minimum (resp. maximum) at $\left[\overline{\mathbf{p}}_{1}, \lambda_{1}\right]$ are

$$
\nabla_{\mathbf{p}} L\left(\mathbf{p}_{1}\right)=0, \nabla_{\lambda} L\left(\lambda_{1}\right)=0,
$$

and

$$
\mathbf{y} t^{H} \nabla_{\mathbf{p}}^{2} L\left(\mathbf{p}_{1}\right) \mathbf{y} t \geq 0,(\text { resp. } \leq 0),
$$

with $\mathbf{y}$ defining the feasible directions $\left\{\mathbf{y} t \mid \nabla_{\mathbf{p}} h\left(\mathbf{p}_{1}\right)^{H} \mathbf{y} t=0\right\}$. Under the unit norm constraint, it can be seen that $\left[e^{-j \theta}, e^{j \theta}, 0, \ldots, 0\right]\left[y_{1}, y_{1}^{*}, \ldots, y_{N}, y_{N}^{*}\right]=0$ is the feasible direction, which constrains $y_{1}^{*}=-y_{1} e^{-j 2 \theta}$ and imposes no constraints on $y_{i}, i=2, \ldots, N$.

Under the assumptions on sources, the first term in Eq. (30) evaluating at $\mathbf{p}_{1}$ can be expressed as

$$
\nabla_{\mathbf{p}} L\left(\mathbf{p}_{1}\right)=\mathbf{E}\left(\begin{array}{c}
0 \\
G^{*}\left(q_{1}^{*} s_{1}\right) g\left(p_{1}^{*} s_{1}\right) s_{1} \\
0 \\
\vdots \\
0
\end{array}\right)+\lambda_{1}\left(\begin{array}{c}
0 \\
p_{1} \\
0 \\
\vdots \\
0
\end{array}\right) .
$$

With respect to Eq. (28), using Eq.(4)-Eq.(7), we can obtain the expression evaluated at $\mathbf{p}_{1}$ as

$$
\nabla_{\mathbf{p}}^{2} L\left(\mathbf{p}_{1}\right)=\mathbf{E}\left(\begin{array}{cccc}
\mathbf{B}_{1} & 0 & \ldots & 0 \\
0 & \mathbf{B}_{2} & \ldots & 0 \\
\vdots & \vdots & \ddots & \vdots \\
0 & 0 & \ldots & \mathbf{B}_{N}
\end{array}\right)+\lambda_{1} \mathbf{I},
$$

where

$$
\mathbf{B}_{i}=\left(\begin{array}{cc}
0 & s_{i}^{2} G^{*}\left(q_{1}^{*} s_{1}\right) g^{\prime}\left(p_{1}^{*} s_{1}\right) \\
0 & 0
\end{array}\right)
$$

and

$$
\frac{\partial^{2} I}{\partial p_{i}^{*} \partial p_{j}}=0 \quad \frac{\partial^{2} I}{\partial p_{i}^{*} \partial p_{j}^{*}}=s_{i} s_{j} G^{*} g^{\prime} \quad \frac{\partial^{2} I}{\partial p_{i} \partial p_{j}}=0 \quad \frac{\partial^{2} I}{\partial p_{i} \partial p_{j}^{*}}=0,
$$

are used.

Substituting $q_{1}=e^{j \theta}$ and setting Eq.(32) to zero, we can obtain

$$
\lambda_{1}=-\mathbf{E}\left\{G^{*}\left(e^{-j \theta} s_{1}\right) g\left(e^{-j \theta} s_{1}\right) s_{1}\right\} e^{-j \theta} .
$$

To find the solution of $\theta$, the nonlinear function $G$ is expanded using MacLaurin series $G(x) \sum_{k} \alpha_{2 k-1} x^{2 k-1}$, where it is assumed that $G$ is an odd function and the $\alpha$ 's are the real-valued function-dependent coefficients. Then, the approximation estimation of Eq. (36) by using third-order MacLaurin series expansion is obtained as following, where the assumptions on sources are used. Because $\lambda_{1}$ is real-valued, the second and third terms in Eq.(37) must be real valued, which implies that $\arg \left(s^{3} s^{*}\right)=2 \theta$. Combing with 
$s^{*} s^{3}=\left(s^{R}-j s^{I}\right)\left(s^{R}+j s^{I}\right)^{3}=\left(s^{R}\right)^{4}-\left(s^{I}\right)^{4}+2 j\left(\left(s^{R}\right)^{3} s^{I}+\left(s^{I}\right)^{3} s^{R}\right), \quad \theta$ in Eq.(13) is obtained as

$$
\begin{aligned}
\lambda_{1}=-E\left\{\left(\sum_{k=1}^{2} \alpha_{2 k-1}\left(e^{-j \theta} s_{1}\right)^{2 k-1}\right)^{*}\left((2 k-1) \sum_{k=1}^{2} \alpha_{2 k-1}\left(e^{-j \theta} s_{1}\right){ }^{2 k-2}\right) s_{1}\right\} e^{-j \theta} \\
=-E\left\{\left(\alpha_{1}\left(e^{-j \theta} s_{1}\right)+\alpha_{3}\left(e^{-j 3 \theta} s_{1}^{3}\right)\right)^{*}\left(\alpha_{1}+3 \alpha_{3}\left(e^{-j 2 \theta} s_{1}^{2}\right)\right) s_{1}\right\} e^{-j \theta} \\
=-E\left\{\alpha_{1}^{2}+3 \alpha_{1} \alpha_{3} e^{-j 2 \theta} s_{1}^{3} s_{1}^{*}+\alpha_{1} \alpha_{3} e^{j 2 \theta} s_{1} s_{1}^{* 3}+3 \alpha_{1}^{3} s_{1}^{3} s_{1}^{* 3}\right\}, \\
\quad \theta=\frac{1}{2}\left(\frac{2 \mathbf{E}\left\{\left(s^{R}\right)^{3} s^{I}+\left(s^{I}\right)^{3} s^{R}\right\}}{\mathbf{E}\left\{\left(s^{R}\right)^{4}-\left(s^{I}\right)^{4}\right\}}\right)+n \pi .
\end{aligned}
$$

To evaluate the condition in Eq.(31) at $\mathbf{p}_{1}$, we substitute $\lambda_{1}$ in Eq. (36) and $\mathbf{B}_{1}$ in Eq.(34) into the previous equations under the constraint $\mathbf{y}_{1}=\left[y_{1},-y_{1} e^{-j 2 \theta}\right]^{T}$. Then, the condition along the dimension $q_{1}$ for a minimum (resp. maximum) is obtained as,

$$
\begin{aligned}
& 2\left(E\left\{\mathrm{~B}_{11}\right\}+\lambda_{1}\right)>E\left\{\mathrm{~B}_{12} e^{-j 2 \theta}+\mathrm{B}_{12}^{*} e^{j 2 \theta}\right\},(\text { resp. }<) \\
& \begin{aligned}
\Rightarrow-2 E\left\{G^{*}\left(e^{-j \theta} s_{1}\right) g\left(e^{-j \theta} s_{1}\right) s_{1}\right\} e^{-j \theta}>E\left\{s_{i}^{2} G^{*}\left(e^{j \theta} s_{1}\right) g^{\prime}\left(e^{j \theta} s_{1}\right) e^{-j 2 \theta}\right. \\
\left.+\left(s_{i}^{2} G^{*}\left(e^{j \theta} s_{1}\right) g^{\prime}\left(e^{j \theta} s_{1}\right)\right)^{*} e^{j 2 \theta}\right\},(\text { resp. }<),
\end{aligned}
\end{aligned}
$$

where $\mathrm{B}_{i j}$ is the $i_{t h}$ row and $j_{t h}$ column of $\mathbf{B}$. Then, we evaluate the condition in Eq. (31) along the other dimensions $\left[p_{2}, p_{2}^{*}, \ldots, p_{N}, p_{N}^{*}\right]$. As shown in [41], evaluating Eq. (31) for stability requires that each $\left(\mathbf{B}_{i}+\lambda_{0} \mathbf{I}\right), i=2, \ldots, N$ be positive definite for a local minimum and negative definite for a local maximum. The eigenvalues eig $\left(\mathbf{B}_{i}+\lambda_{1} \mathbf{I}\right)=B_{11}+\lambda_{1} \pm\left|B_{12}\right|$ are real valued by inspection of the structure of $\mathbf{B}_{i}, i=2, \ldots, N$. Therefore, the conditions along the dimensions $\left[p_{2}, p_{2}^{*}, \ldots, p_{N}, p_{N}^{*}\right]$ for a local minimum (resp. maximum) are obtained as follows:

$$
\begin{aligned}
& B_{11}+\lambda_{1} \pm\left|B_{12}\right|>0,(\text { resp. }<0) \\
& \Rightarrow-\mathbf{E}\left\{G^{*}\left(e^{-j \theta} s_{1}\right) g\left(e^{-j \theta} s_{1}\right) s_{1}\right\} e^{-j \theta} \pm\left|s_{i}^{2} G^{*}\left(e^{j \theta} s_{1}\right) g^{\prime}\left(e^{j \theta} s_{1}\right)\right|>0,(\text { resp. }<0) .
\end{aligned}
$$

Finally, the stability proof of the reference-based negentropy in Eq. (9) has been completed.

\section{References}

[1] I. Akyldiz, W. Su, Y. Sankarasubramaniam, and E. Cayirci, “A survey on sensor networks,” IEEE Commun. Mag., vol. 40, no. 8, pp. 102-114, Aug. 2002. Article (CrossRef Link)

[2] S. A. A. Kumar, K. Ovsthus, and L. M. Kristensen, “An industrial perspective on wireless sensor networks - a survey of requirements, protocols, and challenges,” IEEE Commun. Surveys \& Tutorials, vol. 16, no. 3, pp. 1391-1412, Jan. 2014. Article (CrossRef Link)

[3] J. Medina-Garc' 1a, T. S'anchez-Rodr'iguez, J. A. G. Gal'an, A. Delgado, F. G'omez-Bravo, and R. Jim'enez, “A wireless sensor system for real-time monitoring and fault detection of motor arrays," Sensors, vol. 17, no. 3, pp. 469-480, Feb. 2017. Article (CrossRef Link) 
[4] M. R. M. Kassim, and A. N. Harun, “Applications of WSN in agricultural environment monitoring systems," in Proc. of Proceedings of the International Conference on Information and Communication Technology Convergence (ICTC'16), Jeju, South Korea, pp. 344-349, Oct. 19-21, 2016. Article (CrossRef Link)

[5] X. Yan, H. Cheng, Y. Zhao, W. Yu, H. Huang, and X. Zheng, "Real-time identification of smoldering and flaming combustion phases in forest using a wireless sensor network-based multi-sensor system and artificial neural setwork," Sensors, vol. 16, no. 8, pp. 1228-1237, Aug. 2016. Article (CrossRef Link)

[6] F. Dong, M. Li, X. Gong, H. Li, and F. Gao, "Diversity performance analysis on multiple HAP networks,” Sensors, vol. 15, no. 7, pp. 15398- 15418, Jun. 2015. Article (CrossRef Link)

[7] F. Dong, H. Li, X. Gong, Q. Liu, and J. Wang, "Energy-efficient transmissions for remote wireless sensor networks: An integrated HAP/satellite architecture for emergency scenarios,” Sensors, vol. 15, no. 9, pp. 22266-22290, Sep. 2015. Article (CrossRef Link)

[8] H. Li, H. Yin, X. Gong, F. Dong, B. Ren, Y. He, and J. Wang, "Performance analysis of integrated wireless sensor and multibeam satellite networks under terrestrial interference,” Sensors, vol. 16, no.10, pp. 1711-1719, Oct. 2016. Article (CrossRef Link)

[9] J. Furtak, Z. Zieli'nski, and J. Chudzikiewicz, "Security techniques for the WSN link layer within military IoT," in Proc. of Proceedings of the IEEE 3rd World Forum on Internet of Things (WF-IoT'16), Reston, VA, USA, pp. 233-238, Dec. 2016. Article (CrossRef Link)

[10] A. Alaiad, and L. Zhou, "Patients' adoption of WSN-based smart home healthcare systems: An integrated model of facilitators and barriers,” IEEE Trans. Professional Commun., vol. 60, no. 1, pp. 4-23, Mar. 2017. Article (CrossRef Link)

[11] S. Han, Z. Gong, W. Meng, C. Li, D. Zhang, and W. Tang, "Automatic precision control positioning for wireless sensor network,” IEEE Sens. J., vol. 16, no. 7, pp. 2140-2150. Dec. 2016. Article (CrossRef Link)

[12] W. Ahmed, O. Hasan, and S. Tahar, "Formal reliability analysis of wireless sensor network data transport protocols using HOL," in Proc. of Proceedings of the IEEE 11th International Conference on Wireless and Mobile Computing, Networking and Communications (WiMob'15), Abu Dhabi, United Arab Emirates, 19-21, Oct. 2015, pp.217-224. Article (CrossRef Link)

[13] U. I. Minhas, I. H. Naqvi, S. Qaisar, K. Ali, S. Shahid, and M. A. Aslam, “A WSN for monitoring and event reporting in underground mine environments," IEEE Syst. J., Article (CrossRef Link)

[14] O. Aulov and M. Halem, "Human sensor networks for improved modeling of natural disasters," IEEE Proc., vol. 100, no. 10, pp. 2812- 2823, Jul. 2012. Article (CrossRef Link)

[15] M. Novey and T. Adal1, "On extending the complex fastICA algorithm to noncircular sources," IEEE Trans. Signal Process., vol. 56, no. 5, pp. 2148-2154, Apr. 2008. Article (CrossRef Link)

[16] M. Ashourian, S. Woo and H. Jeong, "Performance evaluation of blind source separation schemes for separating sensor signals in a distributed network," in Proc. of 2017 International Conference on Convergence Information Technology, Gyeongju, South Korea, 21-23 Nov. 2007. Article (CrossRef Link)

[17] A. Bertrand and M. Moonen, "Distributed canonical correlation analysis in wireless sensor networks with application to distributed blind source separation,” IEEE Transactions on Signal Processing, vol. 63, no. 18, pp. 4800-4813, Sept. 2015. Article (CrossRef Link)

[18] H. Chen, J. Feng, and K.T. Chi, "Sensor selection for source extraction in heterogeneous wireless sensor networks,” International Journal of Communication Systems, vol. 23, no. 4, pp. 543-551, Apr. 2010. Article (CrossRef Link)

[19] G. R. Naik, D. K .Kumar, and M. Palaniswami, "Signal processing evaluation of myoelectric sensor placement in low-level gestures: sensitivity analysis using independent component analysis,” Expert Syst., vol. 31, no. 1, pp. 91-99, Feb. 2014. Article (CrossRef Link)

[20] Z. Luo and L. Zhu, "A charrelation matrix-based blind adaptive detector for DS-CDMA systems," Sensors, vol. 13, no. 8, pp. 20152- 20169, Aug. 2015. Article (CrossRef Link)

[21] N. Besic, G. Vasile, J. Chanussot, and S. Stankovic, "Polarimetric incoherent target decomposition by indeppendent component analysis,” IEEE Trans. on Geosciencce and Remote Sensing, vol. 53, no. 3, pp. 1236-1247, Jul. 2015. Article (CrossRef Link) 
[22] G. Pendharkar, G. R. Naik, and H.T. Nguyen, "Using blind source separation on accelerometry data to analyze and distinguish the toe walking gait from normal gait in ITW children,” Biomedical Signal Processing and Control, vol. 13, no. 5, pp. 41-49, Jul. 2014. Article (CrossRef Link)

[23] G. R. Naik, K. G. Baker, and H. T. Nguyen, "Dependence independence measure for posterior and anterior EMG sensors used in simple and complex finger flexion movements: evaluation using SDICA,” IEEE J. Biomed. Health Inform., vol. 19, no. 5, pp. 1689-1696, Jul. 2015. Article (CrossRef Link)

[24] X. Chen, A. Liu, H. Peng, and R. K. Ward, “A preliminary study of muscular artifact cancellation in single-channel EEG,” Sensors, vol. 14, no. 10, pp. 18370-18389, Oct. 2014. Article (CrossRef Link)

[25] X. Cai,; X. Wang, Z. Huang, and F. Wang, "Performance analysis of ICA in sensor array,” Sensors, vol. 16, no. 5, pp. 637-650, May. 2016. Article (CrossRef Link)

[26] G. Tang, G. Luo, W. Zhang, C. Yang, and H. Wang, "Underdetermined blind source separation with variational mode decomposition for compound roller bearing fault signals,” Sensors, vol. 16, no. 6, pp. 897-913, Jun. 2016. Article (CrossRef Link)

[27] S. Qin, J. Guo, and C. Zhu, "Sparse component analysis using timefrequency representations for operational modal analysis,” Sensors, vol. 15, no. 3, pp. 6497-6519, Mar. 2015. Article (CrossRef Link)

[28] L. T. Duarte, J. M. T. Romano, C. Jutten, K. Y. Chumbimuni-Torres, and L. T. Kubota, "Application of blind source separation methods to ion-selective electrode arrays in flow-injection analysis,” IEEE Sens. J., vol. 14, no. 7, pp. 2228-2229, Jul. 2014. Article (CrossRef Link)

[29] N. Mourad, J. P. Reilly and T. Kirubarajan, "Majorization-minimization for blind source separation of sparse sources,” Signal Processing, vol. 131, no. 1, pp. 120-133, Feb. 2017. Article (CrossRef Link)

[30] L. Zheng, M. Lops, and X. Wang, “Adaptive interference removal for uncoordinated radar/communication coexistence,” IEEE Journal of Selected Topics in Signal Processing, vol. 12, no. 1, pp. 45-60, Feb. 2018. Article (CrossRef Link)

[31] Yujie Zhang, Shizhong Zhang, and Rui Qi, "Compressed sensing construction for underdetermined source separation,” Circuits Systems and Signal Processing, vol. 36, no. 2, pp. 4741-4755, Mar. 2017. Article (CrossRef Link)

[32] Guo, S. Huang, Y. Li, and G. R. Naik, "Edge effect elimination in single-mixture blind source separation,” Circuits Syst. Signal Process., vol. 32, no. 5, pp. 2317-2334, Feb. 2013. Article (CrossRef Link)

[33] J. Li, G. Hu, L. Yan, L. Zhao, and L. Li, "De-multiplexing based on complex ICA for mode group diversity multiplexing system,” Opt. Quantum Electron., vol. 47, no. 2, pp. 217-224, Mar. 2015. Article (CrossRef Link)

[34] Y. B. Li, W. Nie, and F. Ye, “A complex mixing matrix estimation algorithm based on single source points,” Circuits Syst. Signal Process., vol. 34, no. 11, pp. 1-15, Mar. 2015. Article (CrossRef Link)

[35] M. Yu, Q. Lin, L. Kuang, X. Gong, and F. Cong, "ICA of full complexvalued fMRI data using phase information of spatial maps,” J. Neurosci. Methods., vol. 249, no. 1, pp. 75-91, Jul. 2015. Article (CrossRef Link)

[36] T. L.Peng, Y. Chen, and Z. L. Liu, “A time-frequency domain blind source separation method for underdetermined instantaneous mixtures,” Circuits Syst. Signal Process., vol. 34, no. 12, pp. 1-13, Dec. 2014. Article (CrossRef Link)

[37] K. P. Yu, K. Yang, and Y. H. Bai, "Estimation of modal parameters using the sparse component analysis based underdetermined blind source separation,” Mech. Syst. Signal Process., vol. 45, no. 2, pp. 302-316, Apr. 2014. Article (CrossRef Link)

[38] X. L. Li and T. Adali, "Blind separation of noncircular correlated sources using gaussian entropy rate,” IEEE Trans. Signal Process., vol. 59, no. 6, pp. 2969-2975, Feb. 2011. Article (CrossRef Link) 
[39] M. Novey and T. Adali, “Complex ICA by negentropy maximization,” IEEE Trans. on Neural Networks, vol. 19, no. 4, pp. 596-609, Feb. 2008. Article (CrossRef Link)

[40] M. Castella and E. Moreau, "New kurtosis optimization schemes for MISO equalization,” IEEE Trans. Signal Process., vol. 60, no. 3, pp. 1319-1330, Dec. 2012. Article (CrossRef Link)

[41] W. Zhao, Y. H. Shen, Z. G. Yuan, D. W. Liu, P. C. Xu, Y. M. Wei, W. Jian, and N. Sha, “A novel method for complex-valued signals in independent component analysis framework," Circuits Syst. and Signal Process., vol. 34, no. 6, pp. 1893-1913, Nov. 2016. Article (CrossRef Link)

[42] V. D. Bos, “A complex gradient and hessian,” Inst. Electr. Eng. Proc.- Image Signal Process., vol. 141, no. 6, pp. 380-382, Dec. 1994. Article (CrossRef Link)

[43] J. F. Cardoso and A. Souloumiac, “Blind beamforming for non-gaussian signals,” Inst. Electr. Eng. Proc.-Radar Signal Process., vol. 140, no. 6, pp. 362-370, Dec. 1993. Article (CrossRef Link)

[44] A. Hyvarinen, "Fast and robust fixed-point algorithms for independent component analysis,” IEEE Trans. on Neural Networks, vol. 10, no. 3, pp. 626-634, May 1999. Article (CrossRef Link) 

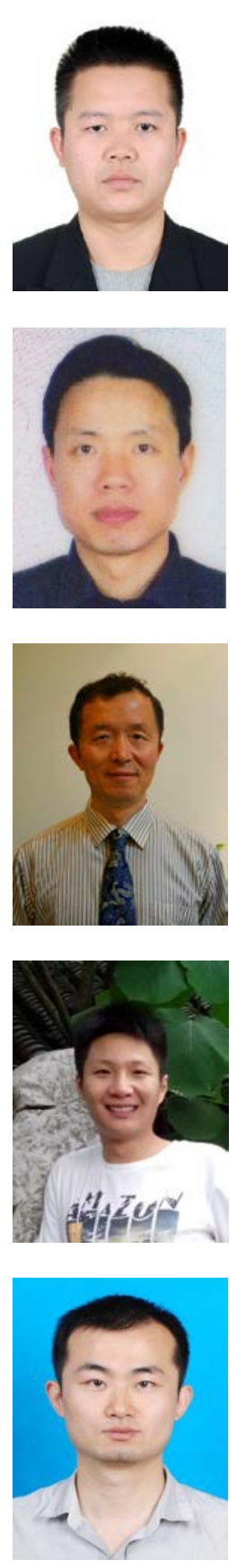

Changliang Deng received the B.S. degree in Communication Engineering and the M.S. degree in Electromagnetic and Microwave Technology from College of Communications Engineering, PLA University of Science and Technology, China, in 2008 and 2013, respectively. He is currently pursuing the Ph.D. degree at Army Engeering University, China. His research interests include blind signal processing, wireless communication, MIMO, and microwave and antenna technology

Yimin Wei received the B.S. degree in wireless communication from College of Communications in 1991, and the Ph.D. degree in wireless communication from PLA University of Science and Technology, China, in 2002, respectively. He is currently a Proferssor of Army Engeering University, China. His research interests include blind signal processing, MIMO, and wireless communication.

Yuehong Shen received the PhD degree in 1999 in Nanjing University of Science and Technology, China. He is currently a professor of Army Engeering University, China. His research interests include wireless MIMO communication, digital signal processing, statistical signal analysis, wireless statistic division multiplexing. He has authored over 50 refereed journal articles and conference papers in these areas.

Wei Zhao received the B.S. degree in Communication Engineering and the Ph.D. degree in Wireless Communication from College of Communications Engineering, PLA University of Science and Technology, China, in 2012 and 2016, respectively. His research interests include blind signal processing, MIMO, and wireless communication.

Hongjun Li received the B.S. degree in Communication Engineering and the Ph.D. degree in Satellite Communication Technology from College of Communications Engineering, PLA University of Science and Technology, China, in 2008 and 2017, respectively. He is currently a research assistant of National Innovation Institute of Defense Technology, Academy of Military Science, China. His current research interests include wireless communications, blind signal processing, MIMO, satellite communications and spatial information networks. 